\title{
Using Real-time Monitoring to Enhance Graduation from Extreme Poverty in Bangladesh
}

\author{
Colin Risner and Vishal Gadhavi
}

\begin{abstract}
The total eradication of extreme poverty is a primary focus of the post-Millennium Development Goal (MDG) agenda. Whilst the term 'graduation' is contentious, in this article it is used to refer to the transition of households or individuals out of extreme poverty. Graduation programmes commonly focus on asset transfer to establish a productive micro-enterprise. To operationalise a 100 per cent graduation creates an imperative for programmes to focus on all of their beneficiaries, in particular the very poorest. The combination of smartphones and internet connectivity provides the building blocks of a system that can track the current status of all programme participants and provide frequent and up-to-date census-level information, enabling timely adaptation of interventions. EEP/Shiree, an extreme poverty reduction programme in Bangladesh, has adopted this approach. This article provides evidence from the roll-out of this system and its potential contribution to the practical realisation of the 'leave no one behind' objective.
\end{abstract}

\section{Introduction}

The challenge of the post-MDG agenda is increasingly framed around the commonly articulated notion of 'leave no one behind' and there is an increasing commitment to the total eradication of extreme poverty as the first of a new set of time-constrained Global Development Goals (UNHLP 2013). But is the goal to 'leave no one behind' meaningful? Can the complete eradication of extreme poverty actually be achieved? For the purposes of this article, the term 'graduation' is used to refer to the sustainable transition of individuals and households out of extreme poverty by whatever means, and 'leave no one behind' means leaving no one in a state of extreme poverty. Hence, 100 per cent graduation and 'leave no one behind' are defined as synonymous.

Programmes designed to graduate people out of extreme poverty through transformative livelihood interventions such as asset or cash transfers, often along with training and community mobilisation, are now commonly termed 'graduation programmes'. These programmes typically report anywhere between 60 per cent and 90 per cent graduation rates yet the attainment of 100 per cent graduation is never claimed;' the remainder are often 'written off' as attrition - in other words they are 'left behind' (CLP 2013; Hashemi and Umaira 2011). The goal of eradicating extreme poverty is not actionable if a significant percentage of people are left behind. This goal therefore necessitates working with the poorest of the poor who are the most entrenched in chronic poverty, and who face multiple barriers, to ensure that they succeed in graduating from extreme poverty. There is a need for a toolset of interventions, programme design and management techniques that will enable enhanced graduation.

\section{Impact for money}

One imperative arising from acceptance of this enhanced graduation objective is having the capability to determine the current status of all participating households. This requires programme implementers to be able to monitor movement towards and beyond a graduation threshold (however defined), whilst also having a set of appropriate interventions that can be delivered to promote sustainable transition out of extreme poverty. Hence, the effectiveness and cost-effectiveness of these interventions could be assessed, with the presumed objective being to 
provide just the appropriate type and minimum value of transfers to each beneficiary required to achieve graduation, an objective that could be summarised as achieving maximum impact for money.

Conceptually, knowledge of the depth, causes and minimal effective solution to the poverty situation facing each individual household would allow an efficient programme to eradicate extreme poverty to be designed and implemented. In practice, of course, the complexity and dynamism of the situation, with people continually being thrust back into extreme poverty through health crises, wars, climatic disasters or other shocks, makes the ultimate objective of zero per cent extreme poverty unattainable. However, in principle at least, the approach required by an efficient 100 per cent graduation programme is known.

This article explores some building blocks towards achieving this objective by reference to a large Department for International Development (DFID), Swiss Agency for Development and Cooperation (SDC) and Government of Bangladesh-supported extreme poverty-focused challenge fund in Bangladesh, the Economic Empowerment of the Poorest, or Shiree programme. ${ }^{2}$ Henceforth, the article will refer to this programme as EEP/Shiree.

An independent study showed that EEP/Shiree has consistently targeted the economically bottom poor 3-4 per cent (Ali 2011). Whilst it is easier to graduate households that are closer to a given threshold, it is a far more difficult task for the people further below the extreme poverty line. The poorest of the poor are the chronic poor who live at the very margins of destitution and exhibit a range of multidimensional deprivations beyond being merely income-poor. This includes many fragmented, often female-headed, households ${ }^{3}$ and disabled and old-aged individuals, all of whom face a high burden of exclusion and marginalisation. ${ }^{4}$ Hence, despite the common characteristic of being extremely poor and the common objective of graduation, the set of circumstances faced by individuals will differ significantly. For many such households, economic empowerment interventions involving asset transfers may not be enough and regular social protection transfers are also needed. Multiplication of income sources to include both earned income and transfer payments can often be a successful strategy at the household level.

Whilst the drive to reduce extreme and moderate poverty in Bangladesh based largely on rapid macroeconomic growth has been successful, in the absence of purposive measures the rate of reduction will slow as the proportion of the chronic poor who make up the extreme poor increases.

EEP/Shiree provides a useful 'test bed' for some of the approaches and tools required to achieve the broader objective of the rapid eradication of extreme poverty from Bangladesh and globally, given that any such macro programme will need to address the same core problems of diversity, scale, resource constraint and rapid change. ${ }^{5}$

\section{The challenge of diversity and scale}

The EEP/Shiree theory of change makes two key assumptions: first, despite the severity and multidimensional nature of their poverty, all households have the 'latent potential' to graduate from extreme poverty; and second, it is possible to deliver many thousands of livelihoods interventions at scale in an efficient manner.

This theory of change is particularly challenging from an operational context since it implies that there is no lower exclusion limit into the programme. In other words, all households, even those without an able-bodied wage earner, with severely disabled members or lacking a stable place of abode (for example, street dwellers) should be considered for inclusion as potential project participants during the selection process. To put it another way, the 'leave no one behind' mantra should be restated as 'leave no extreme poor out' when it comes to operational selection. This differs from several programmes that restrict entry to households with productive members and requires that EEP/Shiree interventions encompass methods other than self-employment through micro-enterprise creation. Alternatives include assets being operated by proxy (for example, where an elderly beneficiary receives a productive asset but this is operated by a relative with income being shared) and, crucially, advocating intensively for improved access to social protection transfers (for example, disability or widow's allowances) as part of a mixed strategy towards household level graduation out of extreme poverty. 
The experience of EEP/Shiree working with over 300,000 extreme poor households in five main regions of Bangladesh reveals considerable variation amongst the client group. ${ }^{6}$ These people face a range of overlapping and mutually reinforcing vulnerabilities and deprivations. These include inherited and acquired (often early in life) health and physical development deficiencies, disability, membership of marginalised or excluded groups, ethnic and gender-based discrimination, geographical remoteness, illiteracy, economic and physical insecurity.

Furthermore, EEP/Shiree's qualitative life history and longitudinal tracking studies (Change Monitoring System-5 (CMS-5)), ${ }^{7}$ show that whilst most households do initiate an upward trajectory out of extreme poverty following project support, usually in the form of asset transfer, this trajectory is peppered with obstacles and challenges. The pathways out of extreme poverty include the diversification and purchasing of assets and increased incomes, and the obstacles are the myriad shocks that can bring about a reverse to these gains, such as the sale of assets to pay for an emergency medical need or the loss of a homestead through river erosion.

\section{Monitoring system requirements}

Such a big, complex and dynamic challenge (given the millions of extremely poor households and the diversity of household situations) demands a revolutionary method of monitoring people, households, interventions and change. It demands a system that enables practitioners to find, target and monitor the most persistent pockets of extreme poverty. The programme Monitoring and Evaluation (M\&E) system, the Change Monitoring System (CMS), enables monitoring of transitional movements out of extreme poverty for households, for sub-projects and for the overall programme. Essential for an operational graduation enhancement strategy (see Section 6), the CMS also enables evaluation of the success of interventions and provides evidence to support accountability.

The variation and numbers of households that EEP/Shiree's sub-projects are engaged with, as well as the challenge fund characteristic of the programme, ${ }^{8}$ dictates a diversity of approach. Hence, there are over 250 different income- generating activities (IGAs), from crab fattening to rickshaw pulling, across the non-governmental organisation (NGO) implemented sub-projects. Understanding possible graduation trajectories requires monitoring tools that can accommodate many possible combinations between specific IGAs and particular household characteristics.

\section{Measurement of graduation}

The headline EEP/Shiree graduation statistic is measured via an annual panel survey using a statistically significant sample of beneficiaries to measure change across a number of criteria (Change Monitoring System-3 (CMS-3)). The graduation line used is an index of multidimensional indicators (for example, income, food security, health) from which a household is deemed 'graduated' if it meets a set number (which differs according to rural and urban settings). The results from four years of panel surveys show a huge increase in graduation rates across the programme, with differing rates for different sub-projects. The average graduation rate (weighted for varying number of households per sub-project) was reported as 90 per cent as of the 2014 survey. ${ }^{9}$

This method of evaluation is typical of large poverty reduction programmes. The collection of baseline data and annual panel surveys requires sufficient time to chart progress and hence impact evaluation of graduation is commonly, though not in the case of EEP/Shiree, a postproject reporting exercise. These post-project analyses and subsequent evaluations can provide evidence of what works and what does not, and hence contribute to the formulation of evidencebased graduation models and recommended interventions.

Sole reliance on a post-project reporting exercise does not, however, meet the need of enhancing graduation through process adaptation during the lifetime of programmes. Furthermore, a given graduation rate calculated on the basis of sample data does not allow practitioners to know who are the graduates and who are not. In the case of EEP/Shiree, CMS-3 data indicates an association between those who have not graduated in any of the four annual panel surveys with features such as female-headedness, old age or disability. However, a sample survey cannot, by definition, pick out actual failing or at-risk households from within a population of many thousands. 


\section{Graduation enhancement strategy}

To recap, the key operational challenge for the development community engaged with graduation programmes is that beyond measuring graduation, how can programmes seek to increase graduation performance and realistically ensure zero writeoffs? Also, how can this be achieved at scale given the diversity exhibited in target areas?

An EEP/Shiree annual external review report highlights another difficulty which is:

in knowing how much movement [out of poverty] is needed because even if it seems like a lot, but it doesn't achieve graduation, the effort has been unsuccessful; yet if the movement continues to be pushed beyond graduation [using programme resources] because graduation can't be identified, the extra effort required to continue that momentum would be unnecessary and could be better used elsewhere (Bene 2012).

This has implications for donor and government resource optimisation and allocation. To achieve the total eradication of extreme poverty means knowing when a household has graduated and redirecting resources elsewhere to other households that struggle or fail to graduate, yet continuing to monitor graduated households so that they can be helped if they fall back - i.e. achieving (graduation) impact for money.

Setting aside conceptual problems with the idea of graduation, and accepting that a formulation of a graduation index can provide a reasonably robust indication of movement out of extreme poverty, graduation can be valuable not only as a tool of measuring impact but as an explicit strategy that can be operationalised. This means that if we know the individual person or household that is failing to prosper and is falling behind, projects can intervene and provide extra targeted support. This is what EEP/Shiree terms a 'graduation enhancement strategy', one that seeks to improve graduation rates. To achieve this one needs to know the status of all beneficiaries engaged with the project, a representative sample will not do.

The operational requirement for an extreme poverty eradication graduation programme is for an information-rich environment based on continuous census monitoring at household level. This system would principally serve as a management tool to guide the interventions, rather than a traditional baseline and endline impact study. Hence, a paradigm shift is needed regarding how we think about monitoring and subsequent feedback mechanisms. M\&E becomes 'MeE' where the extra 'e' denotes experiential learning and continuously asking 'am I doing the right things?' (Pritchett, Samji and Hammer 2011, our emphasis added).

\section{Real-time monitoring of graduation}

Technology, principally through the use of a combination of smartphones and internet connectivity, can be used to monitor the progress of change in household conditions at the field level, with results instantly fed back in near realtime to project managers in a format designed to inform, guide and support management decisions in an approach that is increasingly termed 'adaptive programming' (Vowles 2013). Hence, the term 'graduation' can be applied operationally for evidence-based decision-making in large-scale poverty reduction programmes, enhancing impact and driving the march towards zero extreme poverty.

EEP/Shiree has dealt with this challenge of handling diversity at scale using smartphones and adopting near real-time monitoring to attempt to maximise graduation within an extreme poverty challenge fund. At the core of this operation is the Change Monitoring System-2 (CMS-2), a real-time monitoring system designed to close the feedback loop by providing project managers with monthly updated information about a household's condition using smartphones to survey households at the field level. This is central to the graduation enhancement strategy which is being constructed on four basic pillars.

- The first is a data set that provides continuously updated data at census level about every household.

- The second is a visualisation tool through which this data can be easily accessed and information gleaned about the changes that are happening.

- The third pillar is a set of appropriate interventions to address the situation of individual households (or perhaps entire communities) by assessing what negative impacts and constraints exist. 
- The fourth pillar is the resources needed to provide supplemental support for households that have been identified as failing to prosper, enabling a targeted recovery plan intended to reverse this deterioration and move the household towards graduation.

The principal tool by which this has become possible has been the development of the CMS-2 mobile phone-based census survey, through which $20 \mathrm{EEP} /$ Shiree project partners have been monitoring over 100,000 households every month across Bangladesh and accessing this information through an online visualisation dashboard that is updated in real time. ${ }^{10}$ After trial-and-error and incremental adjustments over three pilot phases, EEP/Shiree deployed a full roll-out of the system towards the end of 2012 with the use of smartphones. CMS-2 is essentially a digitised survey that appears as an application (or 'app') on the smartphone. The survey asks a series of intuitive short multiple-choice questions covering a range of indicators (for example, food habits, change in income and savings, source of main income, etc.) which can be delivered in around ten minutes. The system also enables GPS location of households, photos and voice recording.

Field officers in EEP/Shiree's sub-projects are the principle direct liaison with extreme poor beneficiaries at the community level that provide assistance in the management of IGAs and the coaching and skills training that are key to facilitating upward movement out of extreme poverty. ${ }^{11}$ Every field officer in EEP/Shiree's projects (about 732 in total) has been trained and equipped with a CMS-2 ready smartphone, accessed using a unique username and password, and they conduct the survey on a monthly basis with all beneficiaries currently active in the programme. As the smartphones are internetenabled, completed surveys are uploaded to a central server and processed instantly.

The data from across the country stream into the server and are translated into tracking information accessible via an online visualisation. As a result, it is possible to know the status (either as a composite or by individual indicators) and to observe changes on a monthly basis for every household beneficiary. The data are also cross-referenced with baseline data (Change Monitoring System-1, CMS-1) which provides useful static profiling information about the household demographics and characteristics. This allows data from CMS-2 to be disaggregated by important considerations such as gender, location and type of intervention or transfer received.

CMS-2 allows EEP/Shiree to implement its graduation enhancement strategy by fulfilling the first two crucial parts of the four-pillar strategy a frequently updated data set and a tool through which these data are easily accessible and useable for management purposes. CMS-2 allows EEP/Shiree and its partners the unique ability to track the change of household conditions every month, across NGOs and contexts and enable the identification of households that are failing to prosper or may have encountered a sudden shock. This then allows the consideration of implementing the third and fourth pillars of the strategy which is to provide tailored recovery plans backed up by supplementary resources to struggling households. Furthermore, the data set and visualisation also enable easy identification of success stories ('super graduates') and the potential to learn from and perhaps replicate this success.

\subsection{Case study}

To briefly illustrate the system in use, take the case of a female beneficiary identified for additional support making use of CMS-2 data within an urban sub-project. In this case, the implementing partner NGO team had been looking at data to identify beneficiary households reporting 'begging' as the main source of income in the most recent month. The understanding was that, given that all beneficiaries are engaged with some form of productive employment or small business received earlier as the primary project intervention, anyone reporting 'begging' was likely to be demonstrating the relative failure of this primary intervention.

The team visited a female beneficiary called Feroza living in a small dwelling in Kamrangichar, ${ }^{12}$ one of the largest urban slums in Bangladesh. Feroza was originally the main beneficiary in the household but the project team learned that she had sold her initial small business assets so that her husband could buy a different set of productive assets and be the main income earner as he had not previously been earning (an illustration of the gender dynamics that are a cross-cutting issue affecting the lives of extreme poor households). Further investigation through face-to-face discussion revealed that 
Feroza and her two small children had been abandoned by her husband in recent months and that he had taken all of the family's money and assets (tools for tiling and bricklaying). Feroza could no longer afford to pay rent or feed her children and was relying on begging to survive and hence had fallen back into a state of destitution. The team took the action of developing with her a household recovery plan whereby the project will help Feroza think of and establish a suitable alternative livelihoods strategy.

The case demonstrates that CMS-2 can identify struggling households relatively quickly from which further action can be taken. It also highlights the potential need for social protection transfers and other measures of support essential to keeping Feroza and her family permanently out of extreme poverty. The information system has enabled the rapid identification of need and allowed a timely response that has combined the counselling skills of front-line field staff with supplementary direct transfers in order to, potentially, reverse a slide back down into extreme poverty. The strength of the system is that it sets up a mechanism that can allow this search, diagnostic and restitutive process to be undertaken continually, quickly and at very large scale as part of normal project activity.

\section{Beyond monitoring}

It should be re-emphasised that CMS-2 is not concerned only with narrowly defined project activities. Many of the snapshot survey questions relate to factors that are not part of the direct delivery project intervention. Examples include levels of access to social protection transfers provided by the government, use of public services or source of water and place of defecation. Household recovery plans may encompass measures that extend beyond the scope of the direct asset transfer intervention. Examples include advocating with a union chairman for inclusion of the household in social protection benefits or linking the household to specific health service providers depending on need. CMS-2 provides a rich evidence base to support advocacy, a feature that could easily be expanded in future iterations. The coverage of social protection benefits is already collected and reported, in real time, via the project website. Future examples could include levels of access to core public services such as education or health.
This could underpin accountability of politicians and officials through providing information amenable to appropriate levels of disaggregation (for example, to ward level) or aggregation (for example, at national level) equivalent to the scope of the advocacy effort. Put simply, a national campaign needs national data support, a local campaign needs local data support. Also, the mapping tool included in the visualisation provides a powerful presentation of this data.

Given the potential for such technology to disrupt established methods of project management and impact monitoring, the challenges in rolling out the system have been as much organisational as technical. EEP/Shiree's project partner NGOs, perhaps stuck in the traditional end-of-project impact monitoring mind-set, were initially reluctant to ensure that field officers were actually collecting the monthly data. Additionally, many partners did not fully grasp the system as a feedback loop designed to assist their projects and serve as a management tool. It was unsurprising that initial data collection did not match up with the total number of beneficiaries in the programme but since the roll-out there has been a steady increase in the total completed monthly surveys. It took approximately eight months for monthly data collection to cross the 100,000 mark, which has since been consistently met and represents most households currently active in sub-projects. Training of NGO field staff, M\&E staff and management has been crucial to effective roll-out.

Whilst CMS-2 enables development practitioners to focus on key struggling groups, it is not designed to substitute for the face-to-face interaction between field officers and extreme poor beneficiaries, recognised to have a key role in transforming beneficiaries' lives through skills coaching (Heisey 2013). The operational challenge of roll-out has taken time with emphasis having now shifted to data use for graduation enhancement. Despite the huge volume of data collected, dynamic individual household information is limited to the set of about 30 questions and responses collected from the survey tool within the ten-minute contact time. The data require rigorous analysis, interpretation and supplementation via face-toface consultation in order to translate into effective advisory support at the household level as in the case study. 
There are still many management challenges in utilising data effectively and further attention is required to ensure all data collected are of good quality. The key challenge is to successfully embed processes through which project implementers use the datastream in an investigative and creative manner, helping them to challenge their pre-assumptions of what works and to continuously refine their understanding of the underlying causes of change and the determinants of both failure and success within specific household contexts. CMS-2, in this respect, is only one part of the solution.

With over a year of high-volume data now gathered, the potential for data mining, discovering trends, segments and correlations that will enhance understanding of the dynamics of ascents out of and descents into extreme poverty is an additional benefit beyond the direct operational use of the system. The prospect of using data analysis tools for predictive modelling with regard to poverty risk factors is also enticing, as is the potential to add new poverty analysis applications onto the smartphone platform, and this has already commenced.

\section{Conclusion}

If the global effort to eradicate extreme poverty is to be taken seriously, it will require global commitment of ideas and resources to ensure that no one is actually left behind. This implies massive operational targeting of the extreme poor with graduation-type programmes that are embedded within a larger framework of social protection and public service enhancement and reform. ${ }^{13}$ The term 'graduation' provides a benchmark against which a household can be said to have moved out of extreme poverty. Despite the conceptual and theoretical difficulties associated with this term, the 'arbitrariness' of any selected graduation line or threshold, the concept provides a useful perspective from which focus can be directed to those who are beneath the line, and in particular those who are right at the bottom. Practitioners need to know whether beneficiaries of extreme poverty reduction programmes are succeeding or failing to reach the graduation threshold and to have this knowledge for all those engaged in a programme. Traditional methods of evaluating impact or graduation are inadequate to serve this purpose since they rely on sample data sets, and feedback is too slow to allow adaptive programming to take place.

As large-scale poverty reduction efforts increasingly exhaust the scope for raising people who are close to the extreme poverty threshold (the 'low hanging fruit'), the chronically poor must become the focus, and new solutions will be needed to ensure that these people are not left behind. This will require high frequency censuslevel information about each extreme poor beneficiary household to ensure that we can find those who fail to graduate and provide them with the necessary resources and linkages, including to social protection transfers and other sources of support. Thus, poverty reduction programmes of the future need to be highly adaptive and responsive in order to optimise their focus and free up resources for those who fall behind and fail to graduate out of extreme poverty. From an $\mathrm{M} \& \mathrm{E}$ perspective this cannot be done with a business-as-usual approach, but requires knowing who the chronically extreme poor are, understanding the reasons why they are falling behind, and providing timely feedback and tailored support to address the specific constraints faced by these groups and individuals. Whilst $\mathrm{EEP} /$ Shiree is a large-scale, but nevertheless limited, NGO-implemented programme, the same principles would apply to government-led and government-implemented extreme poverty eradication programmes of national scope.

The formulation, implementation and potential of the EEP/Shiree CMS-2 application demonstrates an attempt to enhance graduation utilising smartphones and internet connectivity to enable development practitioners to identify struggling households with monthly updated household data. This real-time monitoring approach represents a paradigm shift in $\mathrm{M} \& \mathrm{E}$ with significant implications for how we assess, respond and adapt to the changing conditions of households engaged with programme interventions. The organisational challenges of converting data collection into data use for graduation enhancement are ongoing and need further investigation. Overall, CMS-2 presents an example of how potential future national extreme poverty reduction efforts could use realtime, comprehensive in scope, monitoring tools to enhance programme effectiveness and ensure that 'no one is left behind'. 


\section{Notes}

1 Even for the constrained group of individuals that are selected to be in the programme - i.e. some people are included within the programme but 'left behind' because the specific programme intervention fails while others are 'left behind' through being 'left out' of the programme.

2 See www.shiree.org - note 'shiree' means 'steps' in Bangla.

3 Hence, a surprisingly low average number of people per household at baseline of 3.69, which has risen over time as people have become economically empowered and many households have defragmented (for example, through the return of a male household member).

4 For example, a 40-year-old man and his family living in the Haor region of northeast Bangladesh where villages remain isolated by flood during the rainy season and where many migrate seasonally for work, will face a completely different circumstance from a 60 -year-old widow belonging to an isolated indigenous community in the Chittagong Hill Tracts in the southeast part of the country.

5 The Household Income and Expenditure Survey 2010 identifies 17.6 per cent as below the lower poverty line - this exceeds 26 million people. See www-wds.worldbank.org/external/default/ WDSContentServer/WDSP/IB/2012/07/12/000 386194_20120712025531/Rendered/PDF/7095 00WP00PUBL0AndExpenditureSurvey.pdf.

6 The five regions in Bangladesh are the Chittagong Hill Tracts which is politically and economically marginalised, the northeast Haor region which is remote with isolated villages flooded for six months per year, the northwestern region which is prone to seasonal hunger or 'monga' and periodic drought, the southern coastal belt which is extremely vulnerable to climatic shocks including cyclones and tidal surges, and Dhaka's urban slums and streets.

7 The Change Monitoring System (CMS) is EEP/Shiree's integrated Monitoring and
Evaluation system. It has been developed, with refinement and additions, over a period of years since the programme's inception: CMS-1: baseline survey for all beneficiaries from which to monitor change over time; CMS-2: a smartphone-based census survey for frequently updated information of change for each household; GMS-3: annual sample panel survey to provide in-depth socioeconomic and nutritional data allowing an assessment of longer term change and the impact of project interventions; CMS-4: to allow beneficiary groups and implementing NGOs in a smaller cohort of 'innovation projects' to assess the probable causes of changes in their socioeconomic conditions utilising 'most significant change' methodology; CMS-5: to provide a standard format for detailed qualitative investigation at the household level.

8 The selection of sub-projects is a highly competitive process with individual NGO submissions proposing different implementation modalities evaluated by an independent expert panel.

9 Figure taken from unpublished September 2014 report by Mascie-Taylor and Goto (2014).

10 Whilst the total number of beneficiary households enrolled onto the programme has peaked at approximately 300,000 households, the implementing period of projects differs depending on the round of NGO contracts. Hence, when some projects close others may start, so the number of beneficiaries within the implementing period of CMS-2 development was approximately 100,000.

11 The EEP/Shiree programme does not seek to minimise the importance of front-line fieldworkers or face-to-face counselling towards achieving graduation and has sought to enhance and recognise this function in parallel with the roll-out of the CMS system.

12 The names of beneficiaries have been changed for anonymity and data protection reasons.

13 See http://en.manifestofortheextreme poor.com/. 


\section{References}

Ali, Z. (2011) Poverty Thresholds Analysis: Reassessing and Revalidating Quantitative Indicators, http://issuu.com/eep.Shiree/docs/poverty_ thresholds_analysis_-_zulfiqar_ali/1?e= $4460133 / 5887933$ (accessed 23 March 2014)

Bene, T. (2012) 'EEP/Shiree Annual Review Report of Economic Empowerment of the Poorest (EEP)', unpublished report

CLP (2013) Graduation: A CLP Learning Note, Chars Livelihoods Programme, www.clp-bangladesh.org/ pdf/clp\%20graduation\%20learning\%20note.pdf (accessed 23 March 2014)

Hashemi, S.M. and Umaira, W. (2011) New Pathways for the Poorest: The Graduation Model from $B R A C$, CSP Research Report 10, Brighton: Centre for Social Protection, IDS

Heisey, J. (2013) Impact Research and the Role of Coaching in Poverty Reduction, Consultative Group to Assist the Poor (CGAP), www.cgap.org/ blog/impact-research-and-role-coachingpoverty-reduction (accessed 23 March 2014)

Mascie-Taylor, N. and Goto, R. (2014) 'A Quantitative Examination of Factors
Contributing to the Graduation from Extreme Poverty of Bangladeshi Households between March 2010 and March 2014', unpublished report

Pritchett, L.; Samji, S. and Hammer, J. (2011) 'It's All About MeE: Learning in Development Projects through Monitoring ("M"), Experiential Learning ("e") and Impact Evaluation ("E")', keynote paper presented at the Northeast Universities Development Consortium Conference, Yale University, New Haven, 12-13 November 2011

UNHLP (United Nations High Level Panel) (2013) A New Global Partnership: Eradicate Poverty and Transform Economies through Sustainable Development, The Report of the High-Level Panel of Eminent Persons on the Post-2015 Development Agenda, New York NY: United Nations

Vowles, P. (2013) 'Adaptive Programming', DFID Blog, www.dfid.blog.gov.uk (accessed 3 April 2014) 